\title{
Sur le chemin du rite, l'oralité comme technique d'inscription
}

Nadine Wanono

\section{OpenEdition}

1 Journals

Édition électronique

URL : http://journals.openedition.org/span/1656

DOI : 10.4000/span.1656

ISSN : 2268-1558

Éditeur

École pratique des hautes études. Sciences humaines

\section{Édition imprimée}

Date de publication : 1 janvier 2014

Pagination : 151-155.

ISBN : 9782909036434

ISSN : 0294-7080

Référence électronique

Nadine Wanono, «Sur le chemin du rite, l'oralité comme technique d'inscription », Systèmes de pensée en Afrique noire [En ligne], 19 | 2014, mis en ligne le 05 février 2020, consulté le 10 février 2020. URL : http://journals.openedition.org/span/1656 ; DOI : 10.4000/span.1656 


\section{Sur le chemin du rite, l'oralité comme technique d'inscription}

\section{Nadine Wanono}

Ingénieure de recherche CNRS Institut des mondes africains (IMAF)

Témoigner de l'importance de l'oralité dans le cadre des enseignements de Michel Cartry à l'EPHE, me permet de rappeler le plaisir et l'émotion que j'avais lorsqu'à la fin des années 1970 et au début des années 1980, j'assistais à ses cours.

Son enseignement m'a accompagné lors de mes premières missions de terrain. Sa manière de dérouler ses idées, de les présenter, de les démêler, de nous faire participer à l'étude de ses propres matériaux nous a incités à rappeler le caractère exceptionnel de son dire. Michel Cartry instaurait un espace singulier lors de son enseignement et, comme il le précisait en se référant à Mallarmé, "l'acteur du rite parvient par la magie de son geste à planter un décor invisible qui en ôtant de la vue la nudité du lieu institue un autre lieu " et il poursuivait en évoquant la place particulière du devin dans la société gourmantché : «[...] un metteur en scène qui règle les rapports du visible et de l'invisible". Lexercice académique auquel $\mathrm{M}$. Cartry se prêtait volontiers, se métamorphosait en un terrain qui ouvrait des pistes sur lesquelles nous risquions de nous perdre, nouvel espace à la rencontre des liens entre visible et invisible.

Il fallait accepter ce programme sans limite dont les sujets abordaient à travers le prisme du dire des rituels sacrificatoires, des consultations de devins, de la géomancie, la notion de territoire.

\footnotetext{
Michel Cartry, nommé en 1974 directeur d'études à l'École praticue des hautes études (EPHE, $\checkmark$ section, Sciences religieuses), reprenait la chaire "Religions d'Afrique Noire"), créée pour Germaine Dieterlen en 1956.
} 
Invités à parcourir un chemin où recherche, questionnement, doute et curiosité rivalisaient, nous avons appris à questionner nos matériaux, à considérer avec une grande minutie les témoignages, les prières, les chants, les incantations, sujets ordinaires pour tout ethnologue désireux d'entreprendre un terrain.

Mais n'est il pas nécessaire de le rappeler aujourd'hui alors que nos enseignements, nos recherches sont soumises à des critères de productivité, où l'excellence devient une terminologie sélective plus qu'un critère de qualité des travaux. C'est dans cette effervescence, cette aspiration vers des horizons dessinés par la compétitivité qu'il nous a semblé opportun d'apporter notre pierre comme un hommage à l'histoire de son enseignement.

Je me permets de rappeler un échange furtif avec un des nouveaux collègues du laboratoire qui reflète assez bien les réactions que le cours pouvait provoquer. Une fois, dans la salle de cours où enseignait Michel Cartry, ce collègue s'avance pour me demander discrètement : "C'est toujours comme ça "? Oui c'était toujours comme ça!

Comme ça ? Que comprendre derrière ce "ça ", à peine prononcé, indéterminé, quel implicite évoquait-il ? Quinze ans plus tard j'ai eu envie de préciser ce "ça ", de le défendre, de le revendiquer pour sa force, son importance, sa lucidité et cette incroyable énergie qui s'en dégageait.

Michel Cartry s'emparait d'un thème, le sacrifice du Pangolin chez les Lele ou la géomancie chez les Gourmantché, pour le déplier, le déplacer, le situer dans une perspective philosophique, ethnologique, comparatiste. Ses références aux travaux de Marcel Détienne, de Meyer Fortes avec les Tallensi, de Ratray avec les Nankanse ou encore de Lévi-Strauss étaient fréquentes et multiples. Effectivement, il ne s'agissait pas de nous transmettre une méthode, une théorie, mais de nous inciter à questionner, à observer nos matériaux sous tous leurs aspects. Il faisait figure de passeur, celui qui transmet et qui agit son dire.

$\mathrm{Si}_{\mathrm{i}}$ je reprends la notion d'espace, terme cher à M. Cartry, notion et réalité qu'il a abordé si souvent lors- 
qu'il nous expliquait le mot terre ou brousse en milieu gourmantché, il lui était nécessaire de nous déplacer, de nous transporter de la salle de classe à la brousse, dans cette brousse appelée Fuali, "qui n'exprime pas une réalité physique ou géographique, mais plutôt un espace aux limites mouvantes, aux limites variant en fonction du temps... "; il nous emmenait là pour nous mettre dans la position de celui qui est sans repère, avec l'évanouissement des limites...

Parfois, nous pouvions effectivement nous sentir un peu menacés par ces indistinctions, ces questionnements repris et répétés à l'infini. Il s'agissait pour lui de nous amener à reconsidérer toutes les catégories que nous aurions pu prendre pour acquises, à redécouvrir nos matériaux, à insuffler un esprit qui nous permette de déplier les jeux de vérité, avec précision, enthousiasme et humour.

Nous venions tous de milieux différents, et je peux rappeler la présence de notre collègue Mamadou Diabaté ${ }^{2}$ et la mémoire de notre collègue Moustapha Sanogo ${ }^{3}$ qui participaient à ces exercices périlleux de mise à plat. Les références étaient nombreuses et le monde helléniste pénétrait souvent l'enceinte de la salle de classe.

Comment considérer le devin, à quel type de devin pouvions-nous avoir à faire, comment sacrifier un animal, la fonction du couteau? Comment le devin gourmantché posait-il ses questions, le balayage du sable, le tracé des traits? la scarification de la calebasse? Chaque question était débattue, considérée, soupesée, terminologie vernaculaire à l'appui, nous étions entre la brousse... et le village...

Le rythme des cours était saisissant : même si Michel Cartry déposait ses feuilles pour faire mine de les consulter, à chaque séance, immanquablement, il présentait le sujet abordé, s'arrêtait à son énonciation pour nous définir avec une minutie tenace son angle d'observation, les comparatismes possibles entre le monde grec et Mossi. Le plus souvent, une fois qu'il s'était emparé de son sujet il partait dans son raisonnement, trouvait la question juste et allait nous l'exposer sans interruption pendant plus d'une heure. C'était totalement époustouflant! Debout, il arpen-

\footnotetext{
2 Mamadou Diabaté, chercheur associé à «Systèmes de pensée en Afrique noire ».

3 Moustapha Sanogo, chercheur associé à "Systèmes de pensée en Afrique noire "), enseignant à l'Université nationale de Côte d'Ivoire.
} 
${ }^{4}$ Philippe Lourdou, anthropologue-cinéaste, docteur en cinéma. tographie, enseigne le cinéma anthropologique à l'Université Paris.

Ouest Nanterre. tait l'estrade, évoquait, imitait, mimait, traçait les signes au tableau, reprenait la calebasse gravée, dessinait les signes géomantiques. Le temps et l'espace de la salle de classe lui appartenaient, il les investissait sans contrainte, il en était le maître, en toute liberté.

Jean Rouch et Germaine Dieterlen étaient invités régulièrement à exposer leurs derniers travaux, leurs films et recherches sur le Sigui, tout particulièrement.

Quelques années plus tard, alors que j'avais eu la possibilité d'intégrer le laboratoire "Systèmes de Pensées en Afrique noire ", Michel Cartry nous avait invités avec Philippe Lourdou ${ }^{4}$ à venir présenter nos rushes tournés en pays dogon sur l'intronisation et les funérailles du Hogon d'Arou. Ce fut sous son impulsion que nous avions commencé à analyser la construction sonore de certaines séquences du film grâce à une analyse fine des différentes prières et à découvrir des invocations silencieuses ou plus exactement muettes, ou à être confrontés à l'hybridité des registres de certains sons qui relevaient du grognement, de l'onomatopée, de voix oraculaires mais qui ne pouvaient se traduire.

Intéressé et curieux des méthodes d'analyses scénographiques proposées par Claudine de France, c'est avec la plus grande attention et bienveillance qu'il nous demanda d'expliciter les notions de destinataire invisible, ou de non montrable. Là encore alors que bon nombre de collègues avaient du mal, pour ne pas dire refuser implicitement ou catégoriquement à considérer le film comme support d'analyse, Michel Cartry non seulement nous donnait la possibilité de partager nos recherches au sein de son séminaire, mais il était prêt à intégrer ces catégories d'analyse filmique dans le cadre de ses réflexions sur le rituel et le sacrifice, par exemple.

En repensant à ces cours et à la manière si particulière de mettre en partage ses réflexions, $M$. Cartry nous transmettait sa passion, certes, mais au-delà de cela, il nous léguait la valeur qu'il accordait à ses matériaux, à ces mots précieux, ces expressions, à la traduction examinée sous toutes ces 
facettes. De fait, le respect qui se dégageait à l'encontre des données de terrain était infini, il y avait un sens de fragilité et il fallait manier ces mots, ces actions, ces sacrifices avec minutie, une grande délicatesse, beaucoup de soin.

En essayant de restituer la force de cette transmission qui s'élevait dans la salle de l'EPHE et l'ambiance qui se dégageait de ses cours, je désirais rendre compte de la fluidité de cette parole, libre, sans contrainte ni compromis et de l'ancrage qu'elle a pu prendre au sein de nos esprits.

Avec le temps, je dirai que c'est bien durant ces premières années, cet enseignement et cette liberté d'association et de comparaison avec Michel Cartry, que j'ai acquis la nécessité d'être au plus près des matériaux, et de les reconsidérer au fil des années, au point de questionner l'usage de la caméra comme révélateur potentiel d'un réel. En effet, le langage cinématographique rendait compte d'une manière si linéaire des rituels si complexes que je me suis tournée vers les capacités que le numérique offrait pour justement restituer la complexité des réalités considérées.

Je me souviens très précisément lorsque M. Cartry m'incitait à comparer des rituels forts distincts comme les rituels de naissance que j'avais pu filmer, avec des rituels agraires ou des rituels de deuil afin de percevoir et comprendre les similitudes et la continuité qui pouvait se révéler d'un rituel à l'autre. Là effectivement, le cinéma tel que nous pouvions le pratiquer à l'époque touchait les limites imposées par le genre.

Avec le recul, ce fut une chance inouie de pouvoir bénéficier de cet enseignement, qui m'a accompagné tout au long de mon terrain.

Son enseignement, son attachement à la parole restent des témoins d'une méthode de transmission dont on peut évaluer à présent, non seulement la richesse, mais aussi et surtout le décalage face au monde universitaire où la productivité, la rentabilité et l'excellence tentent d'effacer la singularité des réalités partagées avec nos collègues africains. 